\title{
The Protective Effect of Egb 761 Against 3-Nitropropionic Acid-Induced Hearing Loss: The Role of Sirtuin 1
}

\author{
Mun Young Chang ${ }^{1} \cdot$ Jihye Rhee ${ }^{2}$ Shin Hye Kim³ $\cdot$ Young Ho Kim \\ ${ }^{1}$ Department of Otorhinolaryngology-Head and Neck Surgery, Chung-Ang University College of Medicine, Seoul; ${ }^{2}$ Department of \\ Otorhinolaryngology-Head and Neck Surgery, Korea University Ansan Hospital, Ansan; ${ }^{3}$ Department of Otorhinolaryngology-Head and Neck
}

Surgery, Korea University Guro Hospital, Seoul; ${ }^{4}$ Department of Otorhinolaryngology-Head and Neck Surgery, Seoul Metropolitan Government-

Seoul National University Boramae Medical Center, Seoul, Korea

Objectives. Local administration of 3-nitropropionic acid (3-NP) to the inner ear induces sensorineural hearing loss. Several studies have shown the otoprotective effects of ginkgo biloba extract EGb 761. Moreover, EGb 761 has been reported to activate Sirtuin 1 (SIRT1). The present study was designed to investigate whether EGb 761 prevents 3-NPinduced sensorineural hearing loss and determine its effects on the expression of SIRT1.

Methods. Sprague Dawley rats were divided into four experimental groups: control group receiving vehicle of 3-NP, EGb group receiving EGb 761, 3-NP group receiving 3-NP, and EGb+3-NP group receiving EGb 761 and 3-NP. EGb 761 was given orally for 5 days. The 3-NP solution was injected into the tympanum 3 days after the start of EGb 761 administration. The auditory brainstem response was recorded before and after the injection. At 4 weeks after the administration of 3-NP or vehicle of 3-NP, cochleae were harvested, and hematoxylin and eosin staining and immunohistochemistry for SIRT1 antibody were performed.

Results: EGb+3-NP group showed significantly lower threshold shifts than 3-NP group. There was a significant preservation of type II fibrocytes and spiral ganglion cells in EGb+3-NP group than in 3-NP group. In EGb+3-NP group, there was a significantly greater number of SIRT1 immunopositive type II fibrocytes and spiral ganglion cells than in 3-NP group. Calculating the percentage of SIRT1 immunoreactive type II fibrocytes and spiral ganglion cells in viable type II fibrocytes and spiral ganglion cells, respectively, EGb+3-NP group showed significantly higher SIRT1 immunoreactive cells than 3-NP group.

Conclusion. These results suggest that EGb 761 may prevent hearing loss induced by 3-NP in an acute ototoxic animal model, which appears to be related with SIRT1 expression.

Keywords. Cochlea; Ginkgo Biloba Extract; Sirtuin 1; 3-Nitropropionic Acid

\section{INTRODUCTION}

- Received May 31, 2017

Revised July 15, 2017

Accepted August 29, 2017

- Corresponding author: Young Ho Kim

Department of Otorhinolaryngology-Head and Neck Surgery, Seoul

Metropolitan Government-Seoul National University Boramae Medical

Center, 20 Boramae-ro 5-gil, Dongjak-gu, Seoul 07061, Korea

Tel: +82-2-870-2442, Fax: +82-2-870-3863

E-mail: yhkiment@gmail.com
A natural toxin is produced by some fungal species, 3-nitropropionic acid (3-NP), irreversibly inhibits succinate dehydrogenase, which is a major component of the mitochondrial electron transport chain complex II [1,2]. The administration of 3-NP can cause several organ dysfunctions. The mechanisms involved in 3-NP-induced organ damage are the generation of superoxide radicals and proinflammatory cytokines, glutamate-induced toxicity, and apoptosis [3-5]. The former, generation of superoxide

Copyright (C) 2018 by Korean Society of Otorhinolaryngology-Head and Neck Surgery.

This is an open-access article distributed under the terms of the Creative Commons Attribution Non-Commercial License (http://creativecommons.org/licenses/by-nc/4.0)

which permits unrestricted non-commercial use, distribution, and reproduction in any medium, provided the original work is properly cited. 
radicals and proinflammatory cytokines, directly activates nuclear factor- $\kappa \mathrm{B}$, inducing cell death [6,7].

At first, 3-NP has been used to study brain diseases, including Huntington disease, as systemic administration of 3-NP in a rat model was reported to produce selective striatal lesions, showing characteristics of Huntington disease [8]. Then, Hoya et al. [9] reported that local administration of 3-NP to the inner ear of rats induced sensorineural hearing loss (SNHL) in a concentration-dependent manner. This animal model has been used to understand the mechanisms for SNHL, such as age-related hearing loss, noise-induced hearing loss, and idiopathic sudden SNHL that are associated with mitochondrial dysfunction [10,11].

Ginkgo biloba extract EGb 761 is a well-known antioxidant. Several studies have shown the otoprotective effects of EGb 761 $[12,13]$. Moreover, EGb 761 has been reported to activate Sirtuin 1 (SIRT1), an nicotinamide adenine dinucleotide-dependent deacetylase with protective effects against apoptosis caused by oxidative and toxic stress $[7,14,15]$. SIRT1 modulates NF-кB signaling by deacetylating Lys310 of subunit p65 and prevents cell death [16]. In addition, SIRT1 blocks apoptosis through deacetylation of p53 [14,15]. In a study using C57BL/6 mice, SIRT1 was reported to be abundantly expressed in the inner ear, including hair cells, fibrocytes of the spiral ligament, strial intermediate cells, strial marginal cells, and spiral ganglion cells, and decrease as loss of hearing and hair cells progressed [17]. Therefore, it has been suggested that SIRT1 may play a crucial role in the maintenance of cochlear function.

In the present study, we have investigated whether EGb 761 prevents 3-NP-induced SNHL and determined the effects of EGb 761 on SIRT1 expression.

\section{MATERIALS AND METHODS}

\section{Animals}

Seven-week-old male Sprague Dawley rats, with weight ranging from 180 to $222 \mathrm{~g}$, were used for this study. Each animal was acclimatized to the laboratory conditions for 1 week before the start of the experiment. All animal experimental protocols were approved by the Institutional Animal Care and Use Committee of Clinical Research Institute of Seoul National University Hos-

\section{H I G G H L I G G H T S}

- The administration of 3-nitropropionic acid (3-NP) through the tympanic membrane induced sensorineural hearing loss successfully.

- The administration of EGb 761 contributed to hearing protection against 3-NP.

- Three-NP reduced Sirtuin 1 expression in the inner ear, while EGb 761 preserved Sirtuin 1 expression. pital, which is accredited by the Association for the Assessment and Accreditation of Laboratory Animal Care International (IACUC No. 2016-0035).

At the beginning of the experiment, there was no middle ear infection in all rats. Rats were randomly divided into four experimental groups, with six rats in each group: control group receiving vehicle (saline: $\mathrm{pH} 7.4,50 \mu \mathrm{L}$ ) of 3-NP, EGb group receiving EGb 761 (40 mg/kg), 3-NP group receiving 3-NP (500 mM, 50 $\mu \mathrm{L})$, and EGb+3-NP group receiving EGb 761 (40 mg/kg) and 3-NP (500 mM, $50 \mu \mathrm{L})$. EGb 761 was administered orally via gavage for 5 days after the baseline auditory brainstem responses (ABRs) were recorded. The administration of 3-NP or vehicle of 3-NP was performed by an intratympanic injection at 3 days after the baseline ABRs.

\section{Systemic administration of EGb 761}

EGb 761 (Ginexin; SK Chemical, Seoul, Korea) was mixed with water and orally provided at a dose of $40 \mathrm{mg} / \mathrm{kg}$ for 5 days.

\section{3-NP intratympanic injection}

Prior to the injection, each animal was anesthetized with 40 $\mathrm{mg} / \mathrm{kg}$ IM of Zoletil (Zoletil 50; Virbac, Bogota, Columbia) and $10 \mathrm{mg} / \mathrm{kg}$ IM of xylazine (Rompun; Bayer-Korea, Seoul, Korea). At 500 mM, 3-NP (Sigma, St. Louis, MO, USA) was dissolved in saline ( $\mathrm{pH}$ 7.4). A $50 \mu \mathrm{L}$ of 3-NP solution was injected into the left tympanum, using a $1 \mathrm{~mL}$ syringe with a 26-gauge spinal needle 3 days after the administration of EGb 761. The right cochlea was destroyed surgically to prevent cross-hearing during ABR recording, as previously described [9].

\section{Auditory brainstem response measurement}

The hearing of the left ear of all animals was estimated by ABRs before treatment. Animals were anaesthetized with $40 \mathrm{mg} / \mathrm{kg}$ IM of Zoletil and $10 \mathrm{mg} / \mathrm{kg}$ IM of xylazine. All experimental procedures were performed in an acoustically insulated booth. The platinum needle electrodes were placed subcutaneously on the vertex (positive), ipsilateral (negative), and contralateral (ground) ear. The Intelligent Hearing Systems (Miami, FL, USA), employing IHS high-frequency transducers (HFT9911-20-0035) and IHS high-frequency software (ver. 3.30), was used to measure ABRs. Three tone-burst sounds of 8,16 , and $32 \mathrm{kHz}$ were applied as the sound stimuli $(1,562 \mu \mathrm{m}$ duration, CoS shaping, $21 \mathrm{~Hz}$ ). It was measured whether the impedance between the electrodes was less than $2 \mathrm{k} \Omega$ or not, before acquiring the electroencephalography signal. The responses were amplified $(100,000 \times)$ and band pass-filtered (100-1,500 Hz). Then, the responses at each intensity level were averaged for 512 sweeps. The intensity level of stimuli went down from $90 \mathrm{~dB}$ sound pressure level (SPL) to $10 \mathrm{~dB}$ SPL, by $5 \mathrm{~dB}$ SPL. ABR recordings were interpreted by one audiologist, who had no information about the experiment. The lowest intensity level of stimuli that showed a reliable waveform in the ABR trace was deter- 
mined as the hearing level.

Thereafter, EGb 761, 3-NP, or vehicle of 3-NP was given to animals. Further ABR thresholds were measured at 2 and 4 weeks after the administration of 3-NP or vehicle of 3-NP. To analyze the change of hearing levels, we calculated the threshold shift, which was defined as the subtraction of the baseline threshold from each of the posttreatment thresholds. Sequentially, a positive threshold shift meant a deterioration of the hearing level.

\section{Histopathology and immunohistochemistry}

At 4 weeks after the administration of 3-NP or vehicle of 3-NP, animals were anesthetized deeply with Zoletil and xylazine, and sacrificed. The left cochleae were harvested and fixed with $4 \%$ paraformaldehyde in $0.1 \mathrm{M}$ phosphate-buffered saline (PBS). The cochleae were immersed in the fixative overnight at $4^{\circ} \mathrm{C}$ and rinsed in $0.1 \mathrm{M}$ PBS containing $10 \%$ sucrose for 1 hour. They were then decalcified with Calci-Clear Rapid (National Diagnostics, Atlanta, GA, USA) for 6 hours at room temperature and washed with PBS. Lastly, they were rinsed in 0.1 M PBS with $10 \%$ sucrose, $20 \%$ sucrose, and 30\% sucrose for 1 hour at room temperature, in regular order, and embedded with optimum cutting temperature compound (Sakura, Torrance, CA, USA). Then, frozen blocks were cut into $10-\mu \mathrm{m}$-thick sections and stained with hematoxylin and eosin for histopathology.

The basal turn of cochlea was occupied by tissue reaction and inappropriate to count fibrocytes and spiral ganglion cells. Therefore, the second turn of the cochlea was used to count the cells. Type II fibrocytes in the spiral ligament and spiral ganglion cells were counted, using an integrated microscopic counting chamber that delineated the area of interest by a square of 2,500 $\mu \mathrm{m}^{2}$ and $10,000 \mu \mathrm{m}^{2}$, respectively. The average cell counts of type II fibrocytes and spiral ganglion cells from two $\times 2,500 \mu^{2}$ and $10,000 \mu \mathrm{m}^{2}$ of each spiral ligament and spiral ganglion, respectively, were used for statistical analysis.

For immunohistochemistry, the sections were preincubated with the blocking solution ( $0.3 \%$ hydrogen peroxide in PBS) for 5 minutes to block endogenous peroxidase, following PBS washing. After $2 \%$ bovine serum albumin for 30 minutes, the sections were incubated with the primary antibody - rabbit antiSIRT1 antibody (1:500, Sigma)-overnight at $4^{\circ} \mathrm{C}$. Then, the sections were incubated with the secondary antibody-biotinylated sheep anti-rabbit antibody (1:200, Sigma) — at room temperature for 40 minutes. After rinsing with PBS, the sections were incubated with streptavidin-peroxidase (Sigma) at room temperature for 30 minutes. Lastly, diaminobenzidine chromogen was administered for visualization. The control experiments were performed without the primary antibody to confirm the lack of nonspecific binding of the secondary antibody. Sections were counterstained with hematoxylin and viewed using light microscopy. The second turn of the cochlea was used to count immunoreactive cells. Immunoreactive cells were counted in the spiral ligament and spiral ganglion cells, using the aforementioned method. Then, the percentage of immunoreactive cells in viable cells was calculated.

\section{Statistical analysis}

IBM SPSS ver. 21.0 (IBM Corp., Armonk, NY, USA) was used for statistical analyses. ABR threshold shifts were analysed using repeated measures analysis of variance (ANOVA). Post-hoc testing reported the Bonferroni procedure, including the mean difference $(M)$, standard error (SE), and probability $(P)$. Histological analyses were performed using one-way ANOVA. Post-hoc testing reported the Bonferroni procedure, including $M, \mathrm{SE}$, and $P$.

\section{RESULTS}

\section{ABR threshold shifts}

Before the administration of materials, there was no significant difference of ABR thresholds among the four groups. Comparisons of ABR threshold shifts postadministration of materials were made (Fig. 1). The influence of treatment over 4 weeks was explored by performing repeated measures ANOVA with ABR threshold shifts across time as a repeated measure ( 2 and 4
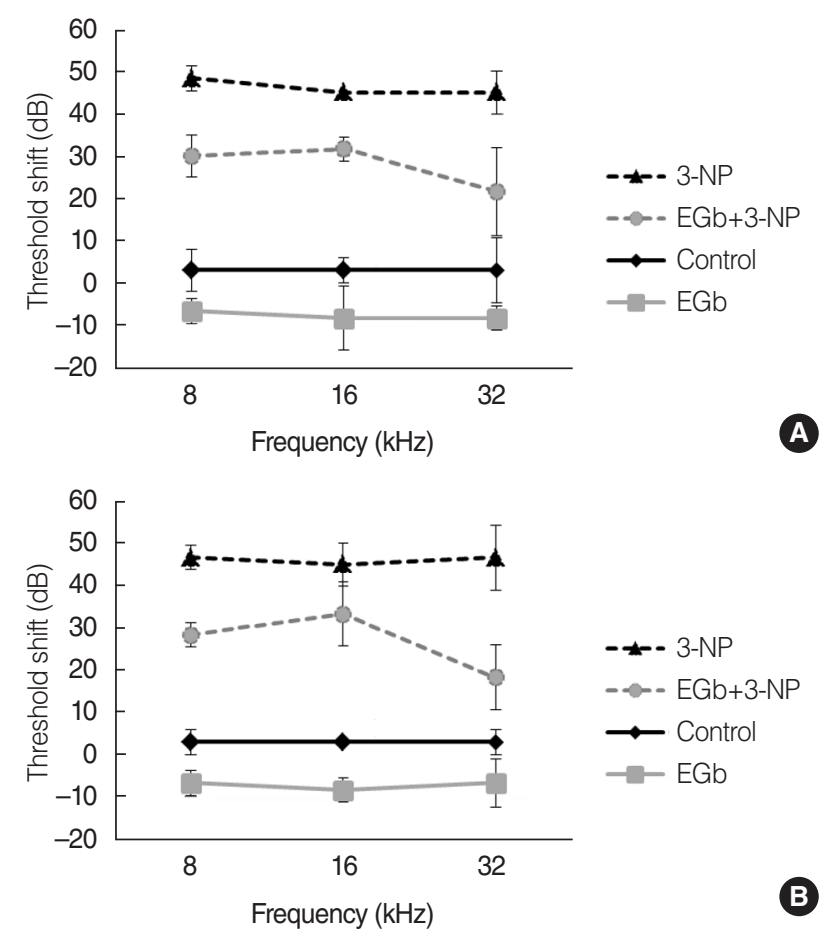

Fig. 1. Threshold shifts in auditory brainstem responses (ABRs) at 2 weeks $(A)$ and 4 weeks (B) after the baseline ABRs and treatment in each group. There was a significant difference of the threshold shifts among the groups $(P<0.001)$. In a comparison of ABRs threshold shifts between EGb+3-NP group and 3-NP group, the threshold shifts in EGb+3-NP group were significantly lower $(P<0.001)$. Error bar is a standard error. 3-NP, 3-nitropropionic acid. 

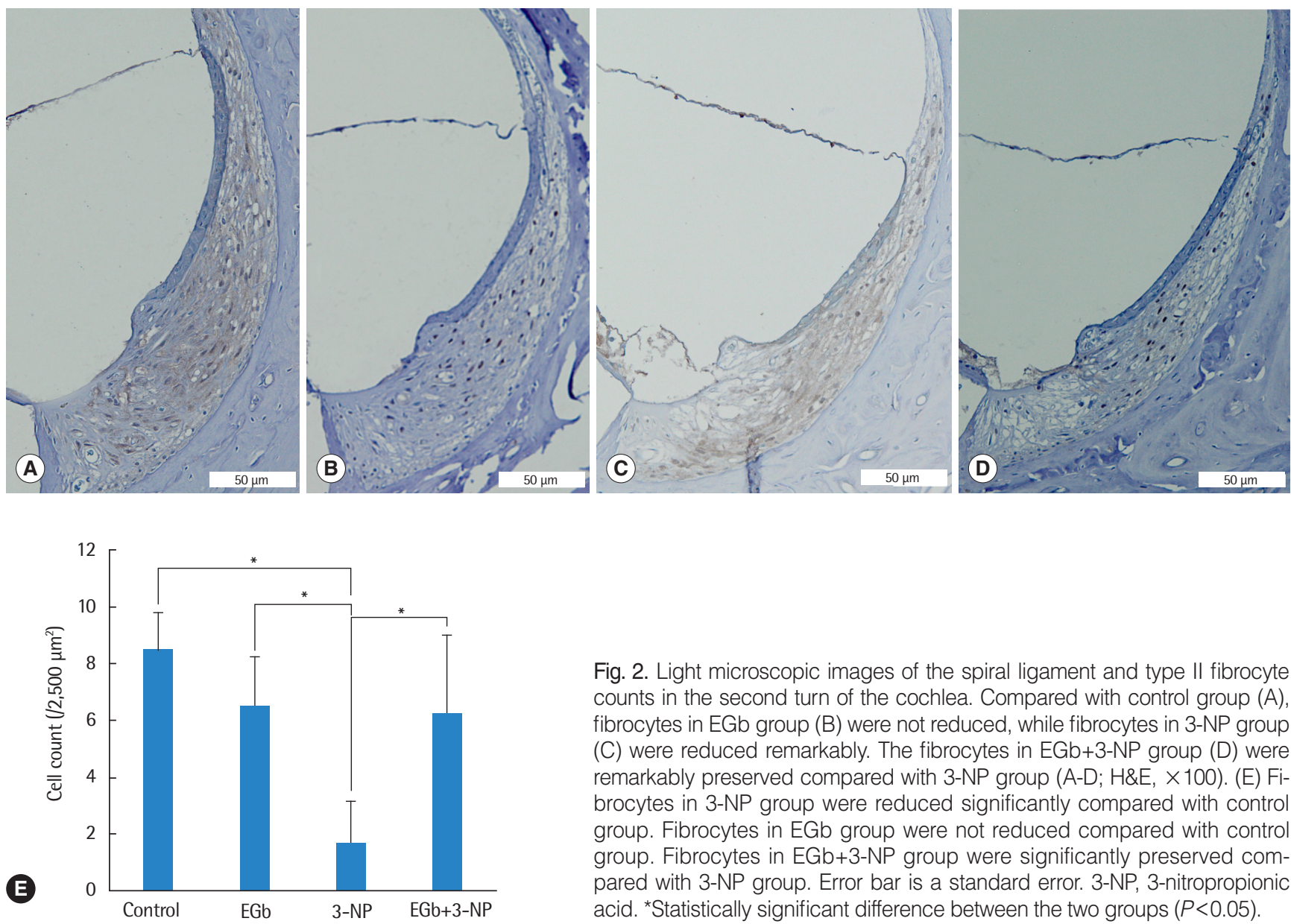

Fig. 2. Light microscopic images of the spiral ligament and type II fibrocyte counts in the second turn of the cochlea. Compared with control group (A), fibrocytes in EGb group (B) were not reduced, while fibrocytes in 3-NP group (C) were reduced remarkably. The fibrocytes in EGb+3-NP group (D) were remarkably preserved compared with 3-NP group (A-D; H\&E, X100). (E) Fibrocytes in 3-NP group were reduced significantly compared with control group. Fibrocytes in EGb group were not reduced compared with control group. Fibrocytes in EGb+3-NP group were significantly preserved compared with 3-NP group. Error bar is a standard error. 3-NP, 3-nitropropionic acid. *Statistically significant difference between the two groups $(P<0.05)$.

weeks) and treatment groups and stimulus frequency as fixed factors. Threshold shifts did not change over time and frequency ( $P=0.364$ and $P=0.261$, respectively). There was a significant difference of threshold shifts among the groups $(P<0.001)$. In a comparison of ABR threshold shifts between control $(M=0.0$, $\mathrm{SE}=0.9 \mathrm{~dB})$ and 3-NP $(M=-46.1, \mathrm{SE}=1.0 \mathrm{~dB})$ group, the latter group showed significantly greater shifts $(P<0.001)$. EGb group $(M=7.5, \mathrm{SE}=0.9 \mathrm{~dB})$ showed significantly lower threshold shifts than control group $(P=0.018)$. In a comparison of ABR threshold shifts between $\mathrm{EGb}+3$-NP and 3-NP group, the threshold shifts in $\mathrm{EGb}+3$-NP group $(M=-27.2, \mathrm{SE}=1.9 \mathrm{~dB})$ were significantly lower $(P<0.001)$.

\section{Histological analysis of type II fibrocytes in the spiral ligament and spiral ganglion cells after 3-NP administration}

Histological comparisons for the spiral ligament and spiral ganglion cells among the groups are shown in Figs. 2-4. There was a significant difference of type II fibrocyte counts $(P<0.001)$ among the groups. In contrast to the normal finding from the spiral ligaments of control group, type II fibrocytes in 3-NP group were reduced remarkably $(M=6.8, \mathrm{SE}=1.2 ; P<0.001)$ with the shrinkage of the spiral ligament and atrophy of the stria vascularis. Type II fibrocytes in EGb group were not reduced compared with control group $(M=2.0, \mathrm{SE}=1.3 ; P=0.895)$. There was a significant preservation of type II fibrocytes in EGb+3-NP group than in 3-NP group ( $M=4.6, \mathrm{SE}=1.2 ; P=0.011)$ (Fig. 2). There was a significant difference of spiral ganglion cell counts among the groups $(P<0.001)$. Spiral ganglion cells were degenerated in 3-NP group compared with control group $(M=20.1$, $\mathrm{SE}=3.2 ; P<0.001)$, similar to the findings in the spiral ligaments. The administration of EGb 761 did not induce the loss of spiral ganglion cells compared with control group $(M=6.5$, $\mathrm{SE}=3.5 ; P=0.504)$. There was a significant preservation of spiral ganglion cells in EGb+3-NP group than in 3-NP group ( $M=9.8$, $\mathrm{SE}=3.2 ; P=0.049)$ (Fig. 3).

\section{Expression of SIRT1 protein in the spiral ligament and spiral ganglion}

Immunohistochemistry was conducted to determine whether SIRT1 protein was present in the spiral ganglion and type II fibrocytes in the spiral ligament (Fig. 4). The average numbers of SIRT1 immunoreactive type II fibrocytes in in control, EGb, 3-NP, and EGb+3-NP groups were 3.9 $\pm 1.7,5.0 \pm 0.9,0.3 \pm 0.5$, and $4.5 \pm 1.2\left(/ 2,500 \mu \mathrm{m}^{2}\right)$, respectively (Fig. 5). Three-NP group 

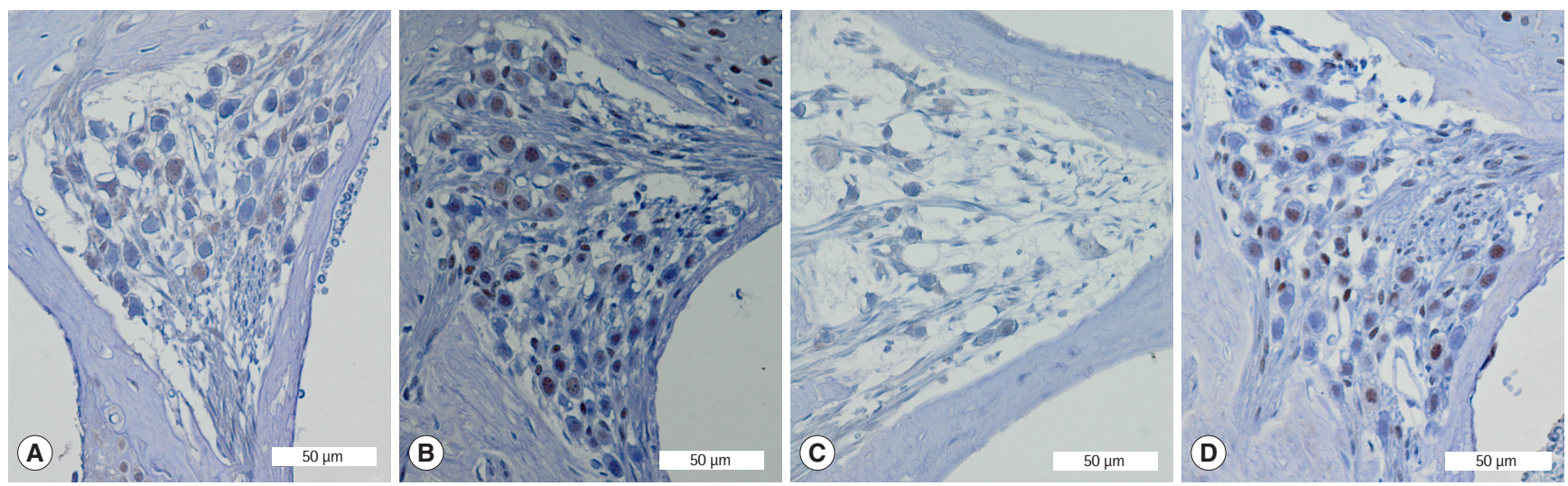
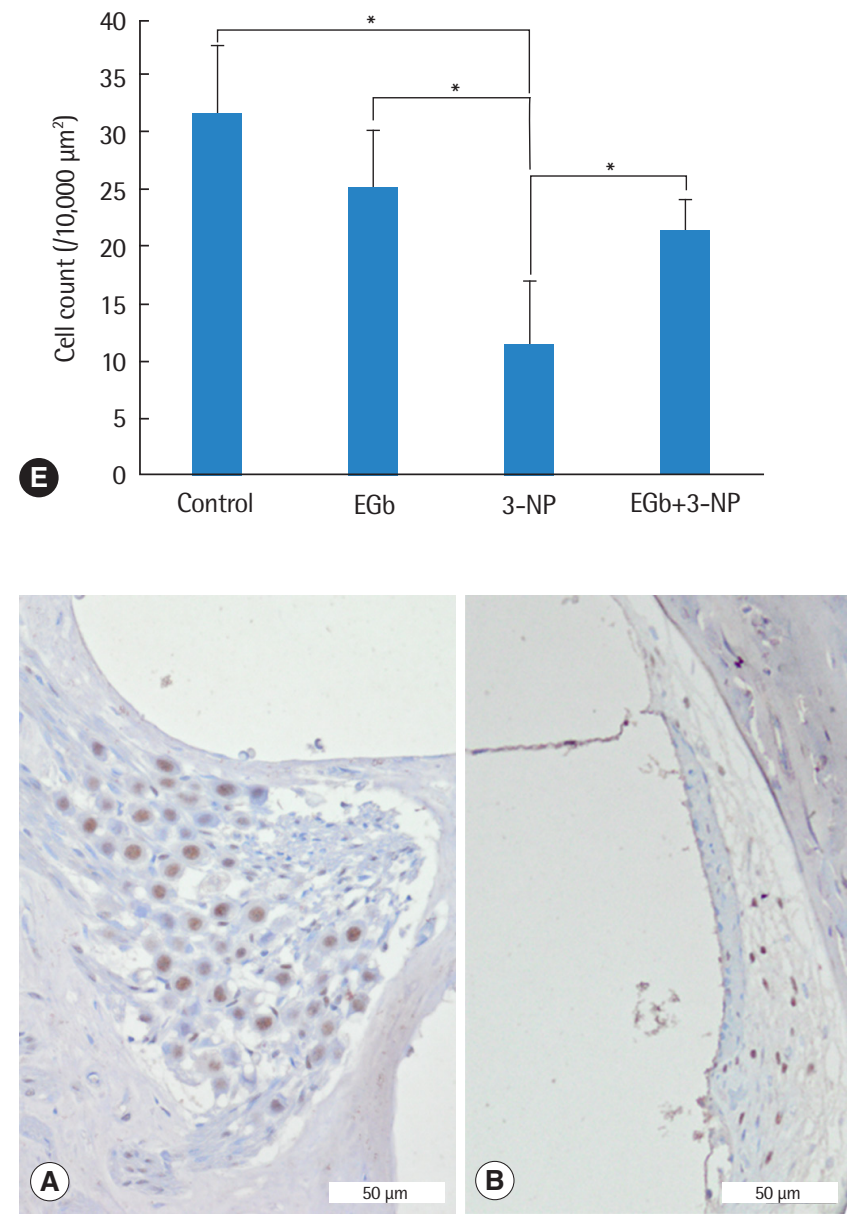

Fig. 4. Representative staining of Sirtuin 1 in the spiral ganglion (A) and spiral ligament (B) from EGb+3-NP group. 3-NP, 3-nitropropionic acid (immunohistochemistry for SIRT1, × 100).

showed significantly lower SIRT1 immunoreactive type II fibrocytes than control, EGb, and EGb+3-NP groups $(M=6.8$, $\mathrm{SE}=1.2, P<0.001 ; M=4.9, \mathrm{SE}=1.2, P=0.007$; and $M=4.6$, $\mathrm{SE}=1.2, P=0.011$, respectively). We calculated the percentage of SIRT1 immunoreactive type II fibrocytes in viable type II fibrocytes. The average percentages of SIRT1 immunoreactive type II
Fig. 3. Light microscopic images of the spiral ganglion and spiral ganglion cell counts in the second turn of the cochlea. Compared with control group (A), spiral ganglion cells in EGb group (B) were not reduced, while spiral ganglion cells in 3-NP group (C) were reduced remarkably. Spiral ganglion cells in EGb+3-NP group (D) were remarkably preserved compared with 3-NP group (A-D; H\&E, X100). (E) Spiral ganglion cells in 3-NP group were reduced significantly compared with control group. Spiral ganglion cells in EGb group were not reduced compared with control group. Spiral ganglion cells in EGb+3-NP group were significantly preserved compared with 3-NP group. Error bar is a standard error. 3-NP, 3-nitropropionic acid. *Statistically significant difference between the two groups $(P<0.05)$.

fibrocytes in control, EGb, 3-NP, and EGb+3-NP groups were $43.1 \% \pm 18.9 \%, 76.9 \% \pm 14.4 \%, 18.0 \% \pm 29.0 \%$, and $72.6 \% \pm$ $21.4 \%$, respectively (Fig. 5). The percentage of SIRT1 immunoreactive type II fibrocytes (\%) in EGb and $\mathrm{EGb}+3-\mathrm{NP}$ groups $(M=58.9, \mathrm{SE}=12.9, P=0.001$ and $M=54.6, \mathrm{SE}=11.1, P<0.001)$ was significantly higher than 3-NP group. The average numbers of SIRT1 immunoreactive spiral ganglion cells in control, EGb, 3-NP, and EGb+3-NP groups were 18.9 $\pm 4.0,22.1 \pm 4.3,4.4 \pm$ 4.1, and 18.7 $\pm 5.0\left(/ 10,000 \mu \mathrm{m}^{2}\right)$, respectively (Fig. 5). In control, EGb, and EGb+3-NP groups, there were significantly more SIRT1 immunoreactive spiral ganglion cells than 3-NP group $(M=14.4, \mathrm{SE}=2.1, P<0.001 ; M=17.7, \mathrm{SE}=2.6, P<0.001$; and $M=14.2, \mathrm{SE}=2.3, P=0.011$, respectively). The average percentages of SIRT1 immunoreactive spiral ganglion cells in control, $\mathrm{EGb}, 3-\mathrm{NP}$, and $\mathrm{EGb}+3$-NP groups were $68.6 \% \pm 14.5 \%$, $81.5 \% \pm 18.4 \%, 38.1 \% \pm 35.1 \%$, and $87.7 \% \pm 26.3 \%$, respectively (Fig. 5). $\mathrm{EGb}+3-\mathrm{NP}$ group $(M=49.6, \mathrm{SE}=13.8 ; P=0.009)$ showed significantly higher percentage of SIRT1 immunoreactive spiral ganglion cells than 3-NP group.

\section{DISCUSSION}

Initially, 3-NP was introduced as a toxin that induced the clinical features of Huntington disease. The main mechanism of 3-NP is 

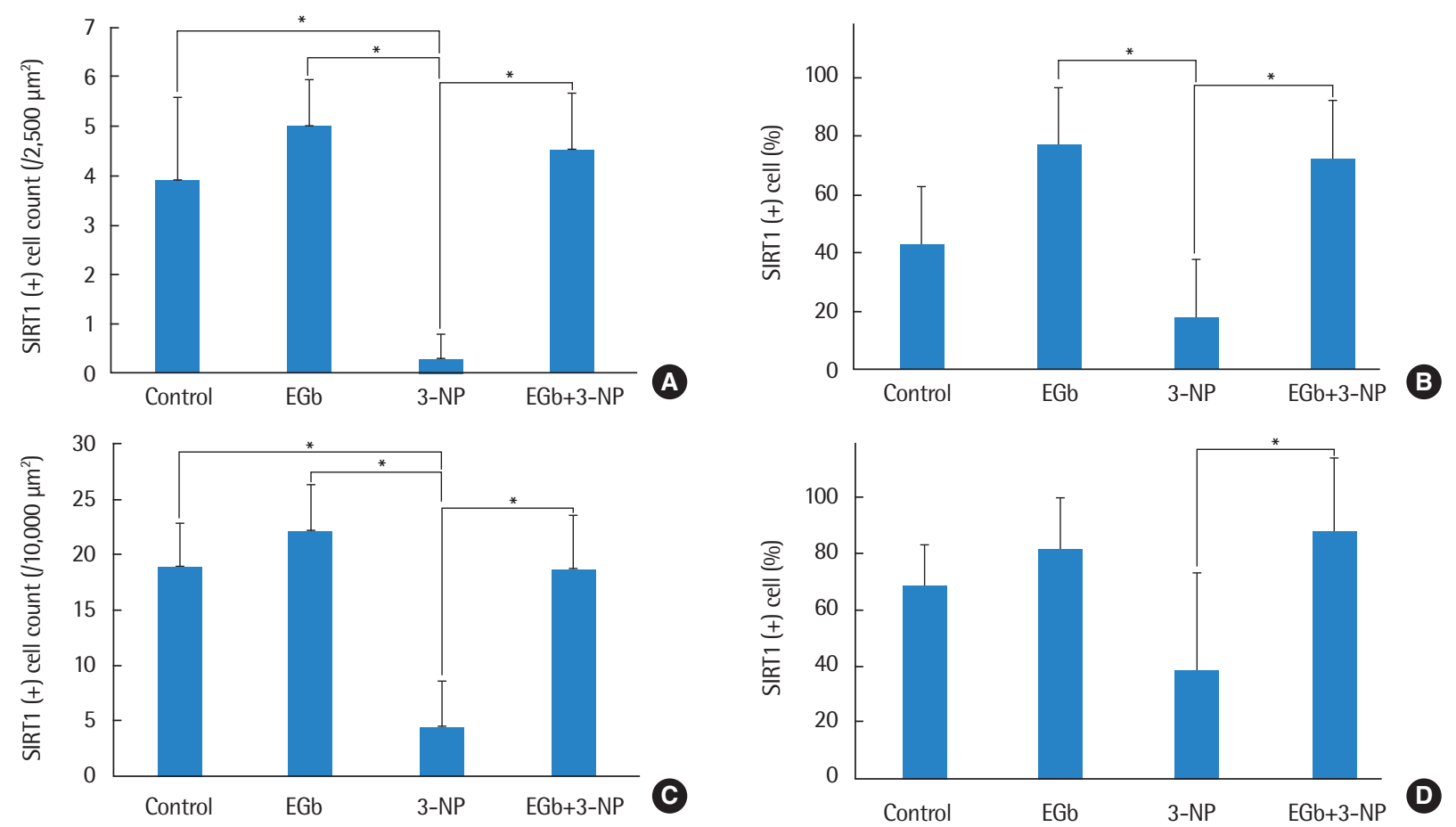

Fig. 5. The mean cell counts (A) and proportion (B) of SIRT1 immunoreactive type II fibrocyte. Three-NP group showed significantly lower SIRT1 immunoreactive type II fibrocytes than control, EGb, and EGb+3-NP groups $(P<0.001, P=0.007$, and $P=0.011$, respectively). The proportion of SIRT1 immunoreactive type II fibrocytes in 3-NP group was significantly lower than EGb and EGb+3-NP groups $(P=0.001$ and $P<0.001$, respectively). The mean cell counts $(\mathrm{C})$ and percentage $(\mathrm{D})$ of SIRT1 immunoreactive spiral ganglion cell. Three-NP group showed significantly lower SIRT1 immunoreactive spiral ganglion cell than control, EGb, and EGb+3-NP groups $(P<0.001, P<0.001$, and $P=0.011$, respectively). The proportion of SIRT1 immunoreactive spiral ganglion cells in 3-NP group was significantly lower than EGb+3-NP group $(P=0.009)$. Error bar is a standard error. SIRT1, Sirtuin 1; 3-NP, 3-nitropropionic acid. *Statistically significant difference between the two groups $(P<0.05)$.

a mitochondrial dysfunction through the inhibition of succinate dehydrogenase, inducing selective striatal pathology in the brain of rodents [8]. In 2004, 3-NP was reported to induce SNHL in rats [9]. Similar to the action in the brain, 3-NP has been established to induce mitochondrial dysfunction via the depletion of mitochondrial ATP in the cochlea, leading to cochlear energy failure and resulting in the degeneration of fibrocytes in the spiral ligament and spiral ganglion cells [9,18]. Fibrocytes and spiral ganglion cells play crucial roles in hearing. In particular, fibrocytes are essential for the maintenance of the endolymphatic potential, which is a fundamental condition for normal hearing [10]. This process requires many active ion channels, transporters, and pumps [10]. Sequentially, fibrocytes contain numerous mitochondria and are vulnerable to 3-NP. The loss of fibrocytes in the spiral ligament has been reported to be the culprit of several types of hearing loss, including age-related and noise-induced hearing loss in a mouse model $[19,20]$. Therefore, several researchers have used a 3-NP-induced hearing loss model to evaluate the mechanism of SNHL and develop otoprotective medicine $[9,11]$. Moreover, in this present study, a 3-NP-induced hearing loss model was used to investigate the otoprotective effects of EGb 761.
Most researchers applied 3-NP into the cochlea by inserting microcannula through the round window or cochleostomy site [9,11]. Although this method can apply an accurate dose of 3-NP, it can cause unnecessary cochlear damage, which can lead to a confusion of the exact effect of 3-NP. Another method was suggested by Tian et al. [18], who applied 3-NP through the tympanic membrane without cochleostomy or round window penetration. Through this method, SNHL was successfully induced without additional cochlear damage. In the present study, we also applied 3-NP through the tympanic membrane. SNHL was successfully induced, and fibrocytes in the spiral ligament and spiral ganglion cells in 3-NP group decreased. The administration of 3-NP through the tympanic membrane seems to be effective in inducing SNHL.

The underlying mechanisms of EGb 761 have been suggested as follows: increase in blood supply by vasodilation, antagonism of platelet activating factor, and antioxidant effects [7,21,22]. In addition to these effects, it has also been reported that EGb 761 stabilizes mitochondrial respiratory chain function [23] and protects against 3-NP-induced brain toxicity [24]. Although the protective effect of EGb 761 against 3-NP-induced hearing loss has not been reported yet, the otoprotective effect of EGb 761 
has been shown under several conditions, including ototoxic drug, noise, and aging [25-27]. Therefore, we hypothesized that EGb 761 had an otoprotective effect against 3-NP-induced hearing loss. In this study, ABR threshold shifts in EGb+3-NP group were significantly lower than in 3-NP group. There has been a significant preservation of fibrocytes in the spiral ligament and spiral ganglion cells in EGb+3-NP group than in 3-NP group. The administration of EGb 761 might contribute to hearing protection against 3-NP through the preservation of fibrocytes and spiral ganglion cells.

The role of SIRT1 was reported as the mechanism of protective effects of EGb761 [7]. Longpre et al. [7] reported that EGb 761 activated SIRT1 in neuronal cells, which deacetylated Lys310 of subunit p65. This leaded to the reduction of NF-kB activity, resulting in the protection of neuronal cells. In addition, SIRT1 expression in the cochlea has been reported to be related with hearing loss $[17,28]$. We focused on SIRT1 among the several molecular mechanisms of EGb 761 against neurotoxicity. SIRT1 is an nicotinamide adenine dinucleotide-dependent deacetylase, which controls mitochondrial biogenesis, inflammatory pathways, and apoptosis [14,15]. At the molecular level, SIRT1 prevents cell death through deacetylation of p65 and p53 $[14,15]$. SIRT1 has been shown to be associated with the prevention of several diseases, such as Alzheimer disease, cancer, and type 2 diabetes [29]. With respect to hearing, SIRT1 is expressed in the inner ear of mice, including hair cells, spiral ganglion cells, and fibrocytes of the spiral ligament. SIRT1 expression is diminished as hearing loss progresses [17,28]. Moreover, it has also been suggested that SIRT1 may play a crucial role in the maintenance of cochlear function $[17,28]$. In this study, SIRT1 expressions in fibrocytes and spiral ganglion in EGb and $\mathrm{EGb}+3-\mathrm{NP}$ groups were significantly higher in than 3-NP group. The percentage of SIRT1 immunoreactive fibrocytes and spiral ganglion over viable cells showed the same tendency. However, there was no significant difference of SIRT1 expressions between control and EGb group. Sequentially, 3-NP appears to reduce SIRT1 expression, while EGb 761 seems to preserve SIRT1 expression, rather than upregulating it in the cochlea. The maintenance of SIRT1 expression by the administration of EGb 761 may contribute to hearing protection in a 3-NP-induced hearing loss model.

The present study had some limitations. First, small numbers of animals were used. Second, the status of cochlear hair cells was investigated with cross-sectional findings of cochleae instead of cochlear surface preparation. Cross-sectional histological study of cochleae permits a better observation of inner and outer hair cells; however, this technique does not enable a quantitative evaluation of cochlear hair cell loss. Nevertheless, cochlear hair cells in EGb+3-NP group showed the tendency to be more preserved than those in 3-NP group. Third, the otoprotective mechanism of EGb 761 was not investigated sufficiently. The significant hearing recovery in the EGb-treated 3-NP group might have resulted from the functional restoration of the fibrocytes in the spiral ligament, spiral ganglion cells, and hair cells in organ of Corti. And, the protection and regeneration of those cells might be one of important rescue methods against hearing loss by mitochondrial dysfunction. Additional studies for intracochlear effect of EGb 761 would reveal the mechanism of otoprotection by EGb 761 in the future.

The systemic application of EGb 761 showed a significant hearing preservation in an acute ototoxic animal model induced by 3-NP. These effects might be attributed to the protection of fibrocytes in the spiral ligament and spiral ganglion cells, which appears to be related with SIRT1 expression. Further studies are needed to investigate roles of SIRT1 in various ototoxic models.

\section{CONFLICT OF INTEREST}

No potential conflict of interest relevant to this article was reported.

\section{ACKNOWLEDGMENTS}

This work was supported by grant (04-2013-0310) provided from SNUH Research Fund and a clinical research grant provided from Seoul National University Boramae Medical Center, Seoul, Republic of Korea.

\section{REFERENCES}

1. Sone M, Hayashi H, Yamamoto H, Hoshino T, Mizushima T, Nakashima T. Upregulation of HSP by geranylgeranylacetone protects the cochlear lateral wall from endotoxin-induced inflammation. Hear Res. 2005 Jun;204(1-2):140-6.

2. Yin HY, Ma XF, Liu F, Xia M, Xu AT. Protective effect of geranylgeranylacetone on cisplatin ototoxicity. Chemotherapy. 2009;55(1):1-5.

3. Brouillet E. The 3-NP model of striatal neurodegeneration. Curr Protoc Neurosci. 2014 Apr;67:9.48.1-14.

4. Khan A, Jamwal S, Bijjem KR, Prakash A, Kumar P. Neuroprotective effect of hemeoxygenase-1/glycogen synthase kinase- $3 \beta$ modulators in 3-nitropropionic acid-induced neurotoxicity in rats. Neuroscience. 2015 Feb;287:66-77.

5. Kumar P, Kumar P, Khan A, Deshmukh R, Lal Sharma P. Role of neurosteroids in experimental 3-nitropropionic acid induced neurotoxicity in rats. Eur J Pharmacol. 2014 Jan;723:38-45.

6. Drew PD, Chavis JA. Inhibition of microglial cell activation by cortisol. Brain Res Bull. 2000 Jul;52(5):391-6.

7. Longpre F, Garneau P, Christen Y, Ramassamy C. Protection by EGb 761 against beta-amyloid-induced neurotoxicity: involvement of NF-kappaB, SIRT1, and MAPKs pathways and inhibition of amyloid fibril formation. Free Radic Biol Med. 2006 Dec;41(12):178194.

8. Beal MF, Brouillet E, Jenkins BG, Ferrante RJ, Kowall NW, Miller JM, et al. Neurochemical and histologic characterization of striatal excitotoxic lesions produced by the mitochondrial toxin 3-nitropropionic acid. J Neurosci. 1993 Oct;13(10):4181-92. 
9. Hoya N, Okamoto Y, Kamiya K, Fujii M, Matsunaga T. A novel animal model of acute cochlear mitochondrial dysfunction. Neuroreport. 2004 Jul;15(10):1597-600.

10. Fujinami Y, Mutai H, Kamiya K, Mizutari K, Fujii M, Matsunaga T. Enhanced expression of C/EBP homologous protein (CHOP) precedes degeneration of fibrocytes in the lateral wall after acute cochlear mitochondrial dysfunction induced by 3-nitropropionic acid. Neurochem Int. 2010 Feb;56(3):487-94.

11. Fujioka M, Okamoto Y, Shinden S, Okano HJ, Okano H, Ogawa K, et al. Pharmacological inhibition of cochlear mitochondrial respiratory chain induces secondary inflammation in the lateral wall: a potential therapeutic target for sensorineural hearing loss. PLoS One. 2014 Mar;9(3):e90089.

12. Koo JW, Chang MY, Yun SC, Kim TS, Kong SK, Chung JW, et al. The efficacy and safety of systemic injection of Ginkgo biloba extract, EGb761, in idiopathic sudden sensorineural hearing loss: a randomized placebo-controlled clinical trial. Eur Arch Otorhinolaryngol. 2016 Sep;273(9):2433-41.

13. Tziridis K, Korn S, Ahlf S, Schulze H. Protective effects of Ginkgo biloba extract EGb 761 against noise trauma-induced hearing loss and tinnitus development. Neural Plast. 2014;2014:427298.

14. Donmez G. The neurobiology of sirtuins and their role in neurodegeneration.Trends Pharmacol Sci. 2012 Sep;33(9):494-501.

15. Yamakuchi M, Lowenstein CJ. MiR-34, SIRT1 and p53: the feedback loop. Cell Cycle. 2009 Mar;8(5):712-5.

16. Yeung F, Hoberg JE, Ramsey CS, Keller MD, Jones DR, Frye RA, et al. Modulation of NF-kappaB-dependent transcription and cell survival by the SIRT1 deacetylase. EMBO J. 2004 Jun;23(12):2369-80.

17. Xiong H, Dai M, Ou Y, Pang J, Yang H, Huang Q, et al. SIRT1 expression in the cochlea and auditory cortex of a mouse model of age-related hearing loss. Exp Gerontol. 2014 Mar;51:8-14.

18. Tian C, Kim YH, Kim YC, Park KT, Kim SW, Kim YJ, et al. Korean red ginseng ameliorates acute 3-nitropropionic acid-induced cochlear damage in mice. Neurotoxicology. 2013 Jan;34:42-50.

19. Hequembourg S, Liberman MC. Spiral ligament pathology: a major aspect of age-related cochlear degeneration in C57BL/6 mice. J Assoc Res Otolaryngol. 2001 Jun;2(2):118-29.

20. Wang Y, Hirose K, Liberman MC. Dynamics of noise-induced cellular injury and repair in the mouse cochlea. J Assoc Res Otolaryngol. 2002 Sep;3(3):248-68.

21. Agarwal L, Pothier DD. Vasodilators and vasoactive substances for idiopathic sudden sensorineural hearing loss. Cochrane Database Syst Rev. 2009 Oct;(4):CD003422.

22. Birks J, Grimley Evans J. Ginkgo biloba for cognitive impairment and dementia. Cochrane Database Syst Rev. 2009 Jan;(1):CD003120.

23. Eckert A, Keil U, Scherping I, Hauptmann S, Muller WE. Stabilization of mitochondrial membrane potential and improvement of neuronal energy metabolism by Ginkgo biloba extract EGb 761. Ann NYAcad Sci. 2005 Nov;1056:474-85.

24. Mahdy HM, Tadros MG, Mohamed MR, Karim AM, Khalifa AE. The effect of Ginkgo biloba extract on 3-nitropropionic acid-induced neurotoxicity in rats. Neurochem Int. 2011 Nov;59(6):770-8.

25. Nevado J, Sanz R, Sanchez-Rodriguez C, Garcia-Berrocal JR, Martin-Sanz E, Gonzalez-Garcia JA, et al. Ginkgo biloba extract (EGb761) protects against aging-related caspase-mediated apoptosis in rat cochlea. Acta Otolaryngol. 2010 Oct;130(10):1101-12.

26. Park SY, Back SA, Kim HL, Kim DK, Yeo SW, Park SN. Renexin as a rescue regimen for noise-induced hearing loss. Noise Health. 2014 Sep-Oct;16(72):257-64.

27. Xu O, Lu H, Li PQ, Zhang X, Lu Z. Effect of combination of Ginkgo leaf extract and deferoxamine in preventing and treating ototoxicity of cisplatin. Zhongguo Zhong Xi Yi Jie He Za Zhi. 2004 Oct;24(10): 915-8.

28. Xiong H, Pang J, Yang H, Dai M, Liu Y, Ou Y, et al. Activation of miR34a/SIRT1/p53 signaling contributes to cochlear hair cell apoptosis: implications for age-related hearing loss. Neurobiol Aging. 2015 Apr; 36(4):1692-701.

29. Revollo JR, Li X. The ways and means that fine tune Sirt1 activity. Trends Biochem Sci. 2013 Mar;38(3):160-7. 